\title{
Handling of deuterium pellets for plasma refuelling
}

\author{
Jensen, P.B.; Andersen, V.
}

Publication date:

1981

Document Version

Publisher's PDF, also known as Version of record

Link back to DTU Orbit

Citation (APA):

Jensen, P. B., \& Andersen, V. (1981). Handling of deuterium pellets for plasma refuelling. Risø National Laboratory. Risø-M No. 2294

\section{General rights}

Copyright and moral rights for the publications made accessible in the public portal are retained by the authors and/or other copyright owners and it is a condition of accessing publications that users recognise and abide by the legal requirements associated with these rights.

- Users may download and print one copy of any publication from the public portal for the purpose of private study or research.

- You may not further distribute the material or use it for any profit-making activity or commercial gain

- You may freely distribute the URL identifying the publication in the public portal

If you believe that this document breaches copyright please contact us providing details, and we will remove access to the work immediately and investigate your claim 
RIS $\varnothing-M-2294$

HANDLING OF DEUTERIUM PELLETS FOR PLASMA REFUELLING

P.B. Jensen and V. Andersen

Association Euratom - Risø National Laboratory

Abstract. The use of a guide tube technique to inject pellets in pellet-plasma experiments is described. The effect of the guide tube on the mass and speed of a slowly moving pellet $(\mathrm{v} \sim 150 \mathrm{~m} / \mathrm{s})$ is negligible. To improve the divergence in trajectories of the pellets on leaving the guide tube a specially formed brass slide has been developed, which improves the aiming accuracy by a factor of 10. A simple method for determining the mass of large pellets by means of a plate capacitor through which the pellets are shot is described. A method for small pellets as well has been investigated. This scheme employs a microwave cavity operating at around $9 \mathrm{GHz}$.

UDC 621.039 .64

December 1981

Risø National Laboratory, DK 4000 Roskilde, Denmark 
ISBN $87-550-0817-8$

ISSN 0418-6435

Risø Repro 1982 
CONTENTS

Page

1. intRodUCtion......................... 5

2. EXPERIMENTAL SET-UP.................... 5

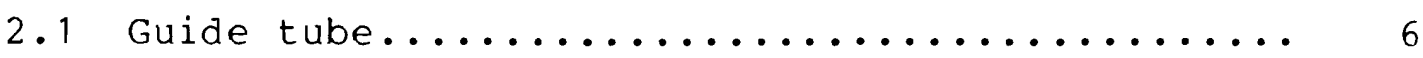

2.2 Angular distribution in pellet injection...... 8

3. DETERMINATION OF PELLET MASS................. 9

3.1 Measuring of the pellet size by a capacitor.... 10

3.2 Measurement of the pellet size by a microwave..

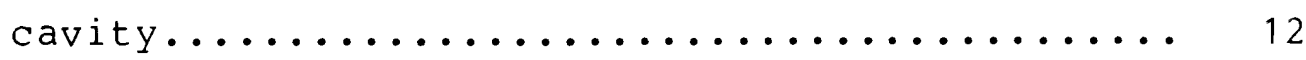

4. CONCLUSION........................... 17

ACKNOWLEDGEMENTS.......................... 18

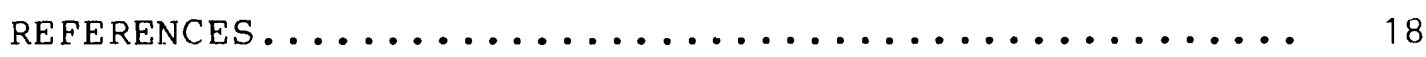

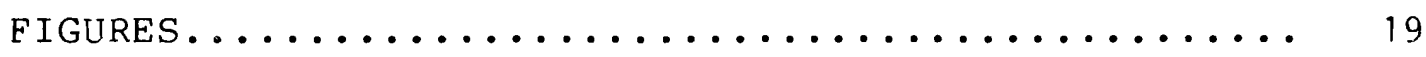




\section{INTRODUCTION}

In the present large tokamak experiments and even more so in future fusion reactors, it is necessary to refuel the plasma, because of its long time of discharge. The two most promising methods at present are gas puffing and injection of cryogenic pellets (Proceedings of the Fusion Fuelling Workshop 1978). The last method has some advantages when compared with the first, presenting the possibility of shaping the plasma density profile to an optimal one. This can be done by choosing the pellet size, injection path, and velocity in an appropriate way. Furthermore, if a divertor is used refuelling deep in the plasma will diminish the problem of removing fresh fuel.

According to existing theory, pellet velocities of up to $10^{4}$ $\mathrm{m} / \mathrm{s}$ may be needed for fusion reactors (Chang et al. 1980), while smaller velocities are sufficient for smaller tokamaks and for experiments in which pellet-plasma interactions are under study (e.g., ISX-B (Milora et al. 1980) and ORMAK (Foster et al. 1977)). In most of the present pellet-plasma interaction experiments the connection between the pellet injector and torus is made by a tube providing a free line-of-sight between the injector and tokamak. This causes the injector-tokamak setup to be rather inflexible in respect to injection port, direction of flight, and time-of-flight. The last point can be important in timing the interaction between the pellet and plasma.

\section{EXPERIMENTAL SET-UP}

The data for the pellets used for injection into the DANTE tokamak for pellet-plasma interaction investigations are the following: The pellets are made of deuterium and are cylindri- 
cal in shape of $0.6 \mathrm{~mm}$ diameter and $2.3 \mathrm{~mm}$ length. This corresponds to $2 \cdot 10^{19}$ molecules. The pellet velocity is $150-200 \mathrm{~m} / \mathrm{s}$. As these pellets are far too large for our plasma (the amount of material in a pellet is around 20 times that in the plasma), a new pellet injector producing smaller pellets has been constructed and is being mounted on the tokamak. The new pellets are also cylindrical, but with both a diameter and length of $0.4 \mathrm{~mm}$. Therefore, in all our development in handling of large pellets, it is important to bear in mind that the technique should be usable for the small pellets as well.

\subsection{Guide tube}

The pellet injector launches the pellets in the horizontal plane (Nordskov et al. 1980). For diagnostic reasons it would be preferable to inject them vertically through the plasma. As the pellet injector is mounted on a cryostat of the helium bath type, it is impossible merely to tilt the injector. Therefore the pellets are launched through a guide tube - a small-bore tube - where no attempt is made to obtain a clear line-of-sight between pellet injector and target. Thus the pellets slide along the tube wall. Different types of guide tubes kept at room temperature have been used. The only preparation was to draw a "Scotch-Brite" through the tube to remove foreign bodies. For a guide tube of around 4-5 m length, the value normally used, the changes in pellet size and velocity were below our measuring uncertainties of about $10 \%$. To determine the influence of the guide tube on the pellet more accurately a $20 \mathrm{~m}$ long guide tube has been set in place. The material for this long tube was brass of inner bore $4 \mathrm{~mm}$. The tube was coiled-up in the horizontal plane with a radius of curvature of approximately $1 \mathrm{~m}$. To be able to measure the velocity of the pellet, glass observation chambers were placed before and after the guide tube. The pellet mass was found following its passage by measuring the pressure increase from the pellet evaporation into a known volume. Averaged over twelve pellets the initial velocity has a mean value and standard deviation of $137 \pm 22 \mathrm{~m} / \mathrm{s}$ and the velocity after passing through the $20-\mathrm{m}$ guide tube was 
$102 \pm 21 \mathrm{~m} / \mathrm{s}$. The ratio of the initial-to-final velocity was $1.38 \pm 0.11$. Under the assumption of a uniform initial pellet size, the pressure measurements indicate that about $30 \%$ of the pellet material either evaporates or is mechanically removed during passage through the tube.

Considering the length of this tube as compared to the $4 \mathrm{~m}$ normally used for the guide tube, the decrease is not very drastic either in pellet size or velocity, and the method is usable for pellets with parameters as given above. At Garching it has been possible to send pellets with velocities of up to $1000 \mathrm{~m} / \mathrm{s}$ through a glass guide tube (Büchl 1980).

Apart from flexibility in the positioning of the pellet injector relative to the tokamak by the use of a guide tube, the method offers other advantages. Several pellet injectors may be connected to the same entrance port by letting the guide tubes merge in a manifold. Thus, the repetition rate may easily be increased for a given injector design. Furthermore, a guide tube offers very good separation between the vacuum systems of the tokamak and pellet injector.

Presently we employ a $5 \mathrm{~m}$ long Teflon tube with an inner bore of $2 \mathrm{~mm}$ and wall thickness of $1 \mathrm{~mm}$. The small dimensions and the material ensure that the tube is very flexible, facilitating changes from one injection port to another. Regarding losses in velocity and mass of the pellet, this tube is apparently similar to the brass tube, but the Teflon tube has several advantages compared with a brass tube of the same size: Firstly, it is not necessary to polish the inside of the Teflon tube, as surfaces are naturally very smooth. Secondly, it is nonconducting, a necessity when using the microwave method mentioned in section 3.2 for determining the pellet size. Finally, the walls are transparent, enabling us to use a pellet detector consisting of an IR LED mounted opposite a photodiode. Hence timing pulses may be obtained anywhere along the guide tube, a useful phenomenon both for velocity measurements of the pellet and for timing the plasma discharge relative to the time of arrival of the pellet. 
For the purpose of demounting, it is convenient to place a valve between the pellet injector and guide tube. This involves a separation of about $10 \mathrm{~cm}$ between the gun barrel and start of the guide tube. To make it easier to enter the guide tube entrance, a funnel is provided for all the guide tubes used, decreasing in diameter from $9 \mathrm{~mm}$ to that of the guide tube itself over a distance of about $40 \mathrm{~mm}$.

\subsection{Angular distribution in pellet injection}

The angular spread in direction of the pellets that leave the guide tube is rather poor, as illustrated in Figure 1. This shows an aluminum foil that has been bombarded with pellets launched from the guide tube. The last $50 \mathrm{~cm}$ of the guide tube were kept straight. The distance between the end of the guide tube and the foil was $60 \mathrm{~cm}$ and the foil was circular with a diameter of $14 \mathrm{~cm}$.

The large spread in pellet trajectories is probably caused by the lack of a well-defined path followed by the pellets through the guide tube. Consequently, if the pellets were forced to move along a predetermined track the divergence would probably be reduced. To test this hypothesis a V-groove was made on the inner side of a brass ring of $6 \mathrm{~cm}$ radius of curvature. The bottom of the groove was rounded to match the shape of the pellet. A $90^{\circ}$ section was cut out of the ring and the guide tube joined tangentially to this section. The joint was made in such a way that the brass termination did not block any part of the guide tube exit. When the pellets were launched by means of this slide, the target foil was marked as shown in Figure 2 .

One observes that in one direction the improvement is very great, whereas in the perpendicular direction no evidence of change is seen. The unimproved direction lies in the plane of the brass circle segment. Obviously, the pellet is released in a rather random fashion. This is probably due to the discontinuity in the radius of curvature of the pellet trajectory at the point of release. If this is the cause it should be possible 
to correct the randomness by changing the radius of curvature smoothly to infinity. This was simply accomplished by bending the exit part of the circle segment backwards so that the radius of curvature changes from $+6 \mathrm{~cm}$ to $-6 \mathrm{~cm}$ over a distance of some centimeters. After this change the final angular distribution of the pellet trajectories is shown in Figure 3. Obviously a drastic improvement has been obtained. Figure 4 shows a photograph of the final version of the slide.

Photographs of the pellet immediately after leaving the slide do not indicate the existence of significant changes in pellet shape. Neither do the dents made in the aluminium foil by the pellet impacts indicate that the pellet has disintegrated during passage of the slide.

The use of a slide as the termination of a guide tube has been tried for the $0.4 \mathrm{~mm} \times 0.4 \mathrm{~mm} \varnothing$ pellets, too. Again, no apparent changes in pellet velocity or mass were observed, but the improvement in divergence was significant.

\section{DETERMINATiON OF PELLET MASS}

When we use the $\mathrm{D}_{2}$-pellets for studying pellet-plasma interactions, it is essential to have a knowledge of the pellet mass prior to its entrance into the plasma. If the pellets produced by the pellet injector were uniform in mass when leaving the guide tube, this knowledge might simply be obtained by collecting a test pellet in a known volume and then measuring the pressure increase when it evaporates. Unfortunately, the pellets do not have the same mass from one shot to another. Hence, it would be very desirable to have a non-destructive method which would be able to determine the actual pellet mass for each shot. 


\subsection{Measuring of the pellet size by a capacitor}

One way of determining the pellet mass is by shooting the pellet through the space between two capacitor plates. As $\varepsilon_{p} \simeq 1.3$ (NBS Technical note 641 1973) for solid $D_{2}$, a small change in capacitance will be observed.

For convenience in the calculations the pellet is assumed to be rectangular with dimensions $a \times b \times c$. The side $a$ is perpendicular to the capacitor plates and the distance between the plates is d. Assuming the capacitor plates are large compared to the size of the pellet and that the pellet lies far from the edges of the plates the field is perpendicular to the pellet surface. Therefore it is necessary to observe only the area of the capacitor covered by the pellet. With no pellet, the capacity of this segment is given by

$$
C_{O}=\frac{\varepsilon_{O} b c}{d}
$$

When the pellet is positioned between the plates the capacity of the same segment is given by

$$
C_{p}=\frac{\varepsilon_{0} \varepsilon_{p} b c}{\varepsilon_{p}(d-a)+a}
$$

Hence the change in capacity is

$$
\Delta C=C_{o}-C_{p}=\frac{V_{p}}{\varepsilon_{p} d^{2}} \frac{\sigma_{o}\left(1-\varepsilon_{p}\right)}{1+\frac{a}{d}\left(\frac{1}{\varepsilon_{p}}-1\right)}
$$

$V_{p}$ being the volume of the pellet.

Because of the small value of $\varepsilon_{p}$, for reasonable values of $\frac{a}{d}$, $\left|\frac{a}{d}\left(\frac{1}{\varepsilon_{p}}-1\right)\right|<1$ is usually satisfied, giving 


$$
\Delta C \simeq \frac{V_{p}}{d^{2}} \varepsilon_{o}\left(\frac{1}{\varepsilon_{p}}-1\right)
$$

$\varepsilon_{p}$ is given by the Clausius-Mossotti relation $M\left(\varepsilon_{p}-1\right) /\left(\varepsilon_{p}+2\right) \rho=$ $\mathrm{P}$, with $\mathrm{M}$ the molecular weight, $\rho$ the density, and $\mathrm{P}$ the molar polarizability. For deuterium $\mathrm{P}$ is $1.99 \mathrm{~cm} / \mathrm{mol}$ (NBS Technical Note 641). To first order this gives $1 / \varepsilon_{p}-1 \simeq-3 P p / M$, which inserted in (3.4) gives

$$
\Delta C \simeq-\frac{3 m_{p} \varepsilon_{O} P}{d^{2} M}
$$

where $m_{p}$ is the pellet mass. Thus to first order the capacitance change depends only on the pellet mass.

If the pellet is tilted relative to the capacitor, it can be subdivided into smaller fragments that can be treated as above. Integration over the whole pellet will then give the same result as (3.5) under the assumption that the dimensions of the complete pellet are small compared with those of the capacitor.

A circuit for determining the change in capacitance is shown in Figure 5 .

$\mathrm{C}_{M}$ is the measuring capacitor, $\mathrm{C}_{C}$ a coupling capacitor, and $\mathrm{A}$ a charge-sensitive amplifier.

The total charge stored on $C_{M}$ and $C_{C}$ is

$$
Q=\left(C_{M}+C_{C}\right) \cdot U
$$

where $U$ is capacitor voltage.

If $R_{S} \cdot\left(C_{M}+C_{C}\right)$ is large compared with the time the pellet is in the capacitor, $Q$ stays constant during the passage of the pellet. That is

$$
0=\Delta Q=\Delta C_{M} \cdot U+\left(C_{M}+C_{C}\right) \cdot \Delta U
$$


The charge transferred to the amplifier is $\Delta Q^{\prime}=C_{C} \cdot \Delta U$

$$
\left|\Delta Q^{\prime}\right|=\frac{\Delta C_{M} \cdot C_{C}}{C_{M}+\bar{C}_{C}^{-}} \cdot U
$$

or for $\mathrm{C}_{\mathrm{C}} \gg \mathrm{C}_{\mathrm{M}}$

$$
\left|\Delta Q^{\prime}\right| \simeq \Delta C_{M} \cdot U
$$

Combining (3.5) and (3.9) one obtains:

$$
\left|\Delta Q^{\prime}\right| \simeq \frac{3 m_{p} \varepsilon_{O} P}{d^{2} M} U
$$

The method has been tried with $d=1 \mathrm{~cm}$ and $\mathrm{U}=400 \mathrm{~V}$. The pellets have a mass of approximately $100 \mu \mathrm{g}$. With $\mathrm{P}=1.99 \mathrm{~cm} / \mathrm{mol}$ the expected $\Delta Q^{\prime}$ is $5.3 \cdot 10^{-15} \mathrm{C}$. The amplifier was an ortec 120 with a specified sensitivity of $4 \mathrm{~V} / \mathrm{pC}$. Thus a pellet should give a signal of around $20 \mathrm{mV}$.

The detected signals were in reasonable agreement with the expected value, but the signal-to-noise ratio was rather poor. This ratio might be improved by increasing $U$, but as the pellet is surrounded by a cloud of evaporated material, the pellet significantly increases the pressure in the capacitor when passing. This may cause a flashover to occur if $U$ is too high, making it impossible to measure properly. In addition, the input stage of the amplifier would very likely burn out. In other words, the utility seems to be restricted to larger pellets, but as we intend to use $0.4 \mathrm{~mm} \times 0.4 \mathrm{~mm} \varnothing$ pellets as well, the method was discarded in favour of a scheme employing a microwave cavity, a technique which has been successfully used to determine the masses of liquid droplets (Lee and Ajmera 1978).

\subsection{Measurement of the pellet size by a microwave cavity}

The microwave cavity we had at our disposal was cylindrical of $26 \mathrm{~mm}$ diameter and $35.5 \mathrm{~mm}$ length. This showed a strong resonance at $8.8 \mathrm{GHz}$. On comparing this with a mode chart for a cir- 
cular cylindrical cavity, the mode was identified as the $\mathrm{TM}_{0} 10$. The electrical field in the cavity for this mode is (collin $1966)$

$$
\begin{aligned}
& E_{Y}=0 \\
& E_{\phi}=0 \\
& E_{Z}=A \cdot J_{O}\left(\frac{P_{01} \cdot r}{a}\right)
\end{aligned}
$$

$A$ is a constant, $J_{O}$ the Bessel function of first kind and $0^{\prime}$ th order, $\mathrm{P}_{01}$ the first zero of this function, and a the radius of the cavity.

The energy content of the electric field in the cavity is given by

$$
w=\text { const. } \varepsilon_{0} \int_{r=0}^{a} \int_{\phi=0}^{2 \pi} \int_{z=0}^{d} E_{z}^{2} r d \phi d r d z
$$

where $d$ is the length of the cylinder.

By inserting $(3.11)$ into $(3.12)$ one gets

$$
W=\text { const.' } \varepsilon_{O} a \pi a^{2} J_{1}^{2}\left(P_{01}\right)
$$

If a small, cylindrical pellet is placed coaxially in the cavity, as illustrated in Figure 6 , the electrical field energy is changed. The total change is

$$
\begin{aligned}
\Delta W=\text { const. } & \left\{\varepsilon_{O} \int_{r=0}^{r} p \int_{\phi=0}^{2 \pi} \int_{z=0}^{d-h} \frac{E_{z}^{2}}{\left(1-\frac{\left.h\left(1-\frac{1}{d}\right)_{p}\right)^{2}}{\varepsilon_{p}} d \phi d r d z\right.}\right. \\
& +\varepsilon_{O}{ }^{\varepsilon} p \\
& \int_{r=0}^{r} p \int_{\phi=0}^{2 \pi} \int_{z=0}^{h} \frac{E_{z}^{2}}{\varepsilon_{p}^{2}\left(1-\frac{h}{d}\left(1-\frac{1}{\varepsilon_{p}}\right)\right)^{2}} r d \phi d r d z
\end{aligned}
$$




$$
\left.-\varepsilon_{0} \int_{r=0}^{r_{p}} \int_{\phi=0}^{2 \pi} \int_{z=0}^{d} E_{z}^{2} r d \phi d r d z\right\}
$$

For small values of $x, J_{1}^{2}(x)+J_{0}^{2}(x) \simeq 1$.

Furthermore assuming $\frac{h}{d}\left(1-\frac{1}{\varepsilon_{p}}\right)<1$

$$
\Delta W \simeq \text { const.' } \cdot\left(1-\frac{1}{\varepsilon_{p}}\right) \varepsilon_{o} V_{p} \text { with } V_{p} \text { the pellet volume.(3.15) }
$$

By combining (3.13) and (3.15), one obtains

$$
\frac{\Delta W}{W} \simeq 3.71\left(1-\frac{1}{\varepsilon_{p}}\right) \frac{V_{p}}{V_{C}}
$$

where $V_{C}$ is the volume of the cavity.

By use of the Clausius-Mossotti relation one obtains

$$
\frac{\Delta W}{\bar{W}} \simeq 11.1 \frac{\mathrm{P} \mathrm{mp}}{\overline{M V_{C}}}
$$

The selectivity factor, Q, of the cavity is defined as follows:

$$
Q=\frac{\omega \cdot W}{\mathrm{P}_{\ell}}
$$

here $w$ is the resonance frequency, $w$ the stored energy in the cavity, and $P_{\ell}$ the power loss.

If $Q$ and $P_{\ell}$ are assumed to be unchanged by the introduction of the pellet, the shift in resonance frequency will be given by 


$$
\left|\frac{d \omega}{d W}\right|=\frac{Q P_{\ell}}{W^{2}} \text { giving }|\Delta \omega| \simeq \omega_{O} \frac{\Delta W}{W} \text {. }
$$

on inserting $(3.16)$ this gives

$$
|\Delta \omega| \simeq 11.1 \omega_{0} \frac{P}{M V_{C}} m_{p}
$$

The frequency shift may be detected with the set-up shown in Figure 7 .

Osc is a tunable microwave generator, D.C. a directional coupler by means of which the impedance mismatch between the oscillator and cavity may be measured. When the oscillator is tuned to the resonance frequency of the cavity these are perfectly matched and no power is reflected from the cavity. As the oscillator is gradually mistuned, more and more is reflected. Hence, the amount of reflected power is a measure of the difference between oscillator and resonance frequency. By mistuning the oscillator slightly, a linear relationship between output voltage and frequency shift is obtained for changes in frequency that are small compared with the resonance frequency.

To introduce the pellet into the cavity two holes are drilled through the cavity end-plates. The guide tube may pass through these holes and the pellet can thereby be passed along the cavity axis. Obviously, this works only when using a non-conducting guide tube.

The cavity we have used employs a loop coupling and is sketched in Figure 8 .

The resonance condition for the cavity is

$$
\mathrm{k}_{\mathrm{O}}=\frac{\mathrm{P}_{0} 1}{\mathrm{a}}
$$

where $k_{O}$ is the wavenumber for the $\mathrm{TM}_{010}$ mode. 
For the cavity shown in Figure 8 this gives a resonance frequency $\omega_{0} / 2 \pi=8.8 \mathrm{GHz}$. With $\mathrm{m}_{\mathrm{p}}=100 \mu \mathrm{g}, \mathrm{V}_{\mathrm{C}}=19 \mathrm{~cm}^{3}$, and $P=1.99 \mathrm{~cm}^{3} / \mathrm{mol}$, equation (3.19) gives $\Delta \omega / 2 \pi=0.3 \mathrm{MHz}$.

The method has been tried with a very makeshift arrangement. In particular, the cavity was in no way optimized and its Q-factor was only about 1500. Yet despite this, clear signals were obtained. For a $100 \mu \mathrm{g}$ pellet, we had a SNR of $25 \mathrm{~dB}$.

Clearly a higher value of $Q$ will give a higher sensitivity. On the other hand the response will be non-linear if $Q$ is too high. An estimate of the highest $Q$ for the $100 \mathrm{\mu g}$ pellet may be found from the following expression for $Q$ :

$$
Q=\frac{\omega_{O}}{2 \Delta \omega}
$$

where $2 \Delta \omega$ is the full bandwidth at half maximum. If $\Delta \omega$ is taken to be $2 \pi \cdot 0.3 \mathrm{MHz}$ this results in a $Q$ value of 15000 , which is not unreasonable for a microwave cavity. Thus, a sufficiently high signal-to-noise ratio should then be easily obtained.

Results from the microwave system for pellets of solid deuterium is shown in Figure 9. To get a reference, the pellets were shot into a microphone. As the microphone responds with a signal proportional to the momentum of the pellet, the signal from the microphone and pellet velocity together determine the pellet mass.

The large spread in data is due to the microphone, because its sensitivity is very dependent on the point of impact. Thus, to check the cavity measurement more carefully, small pieces of plastic were dumped through the cavity. The masses could be determined precisely by weighing.

Test particles of different lengths and thicknesses were used. The results shown in Figure 10 indicate a fairly linear dependence as expected from equation (3.19). 
The reasonably high signal-to-noise ratio for the pellets used, together with the possibility of increasing the selectivity factor by nearly an order of magnitude, indicate the usefulness of the method for the small pellets $(0.4 \mathrm{~mm} \times 0.4 \mathrm{~mm}$ ) as well.

\section{CONCLUSION}

One of the important determinations in the pellet-plasma interaction experiments involves the measurement of the amount of material ablated from the pellet penetrating into the hot plasma. In these studies it is important to know the actual size of the incoming pellet, for comparison with the amount of material ablated, rather than use an average pellet size. For this purpose the microwave cavity measurement is very well suited to a large range of pellet sizes. Furthermore, the method can be used anywhere on the guide tube to test a possible change in pellet size during the flight through the tube. In case the pellet is sufficiently large to penetrate the plasma completely, a measurement of the size of the pellet that remains will be important in order to compare the initial size with the amount of ablation. Because of the high precision of pellet injection into the tokamak by using the guide tube and the slide discussed in section 2.2, it is possible to enter an exit port in the tokamak opposing the entrance port. By use of a suitable valve the remaining pellet can be transferred to a known volume and the pellet size can be determined by the pressure rise in this volume.

Regarding measurements of the pellet size our aim has been to check experimentally and to render probable by calculation that the masses of pellets of solid deuterium is proportional with the signals from capacitors or microwave cavities passes by the pellets. We have not gone into details of absolute measurements, because we intend in practice to make experimental calibrations of the set-ups. 
ACKNOWLEDGEMENTS

The authors thank H. Sørensen for delivering the pellet injector, P. Andersen for excellent technical support, S.A. Andersen for valuable discussions, and P. Michelsen for loan of the microwave equipment.

REFERENCES

BUCHL, K. (1980). Private communication.

CHANG, C.T. et al. (1980). Nuclear Fusion 20, 859-893. COLLIN, R.E. (1966). Foundations for Microwave Engineering (McGraw-Hill Kogakusha, Ltd.).

Foster, C.A. et al. (1977). Nuclear Fusion 17, 1067-1075. LEE, C.S. and AJMERA, R.C. (1978). J. Phys. E (SCi. Instrum.) $11,391-392$.

MILORA, S.L. et al. (1980). Nuclear Fusion 20, 1491-1514. NBS Technical note 641. Survey of the properties of Hydrogen Isotopes below their Critical Temperature. CON-75-10281. NORDSKOV, A. et al. (1980). Risø-M-2245. PROCEEDINGS of the Fusion Fuelling Workshop (1978). Princeton University, Princeton, Nov. 1977, Conf-771129. 


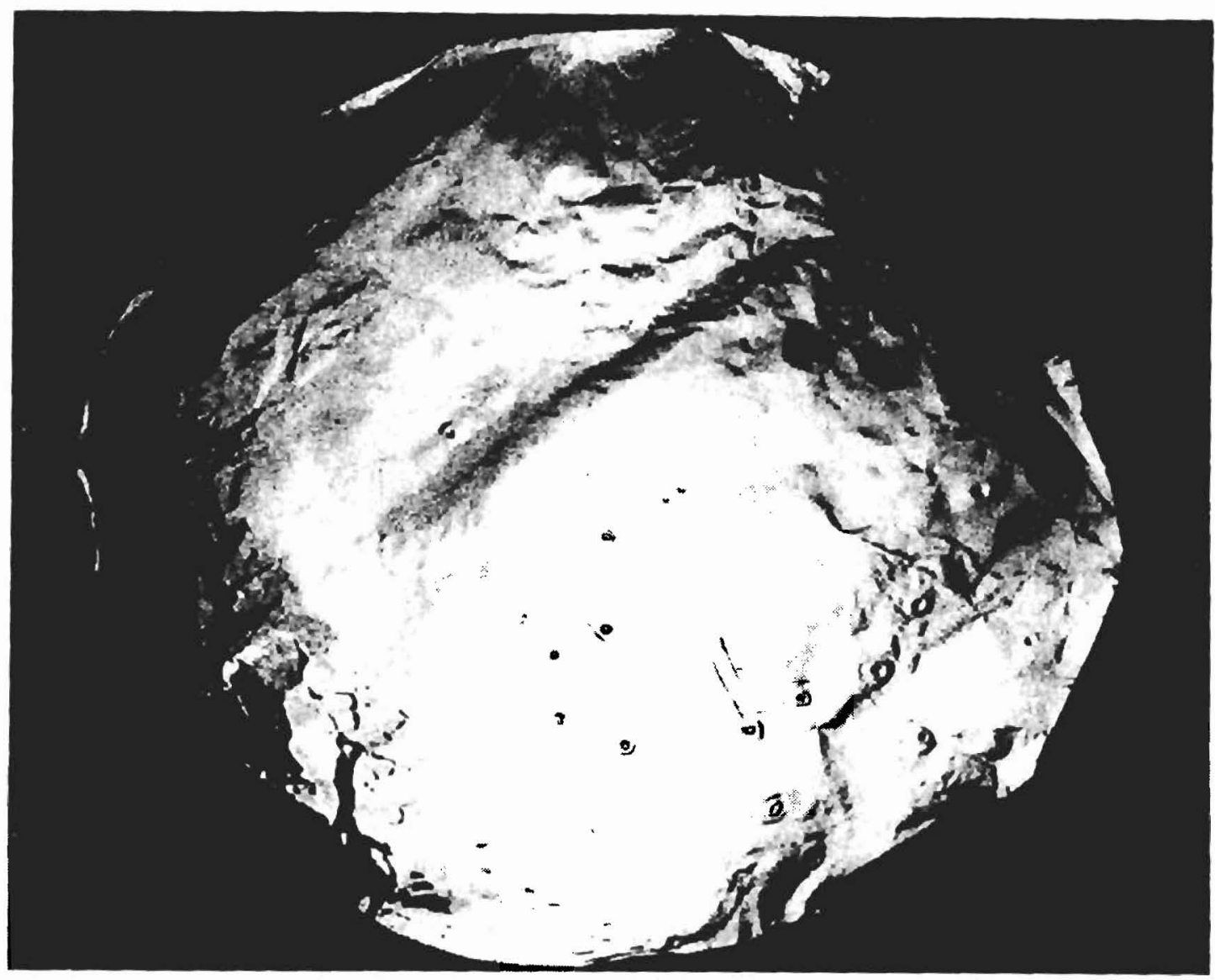

Fig. 1. Target foil showing angular spread in pellet direction on leaving the bare guide tube. The distance between the end of the guide tube and the foil was $60 \mathrm{~cm}$ and the foil was circular with a diameter of $14 \mathrm{~cm}$. 


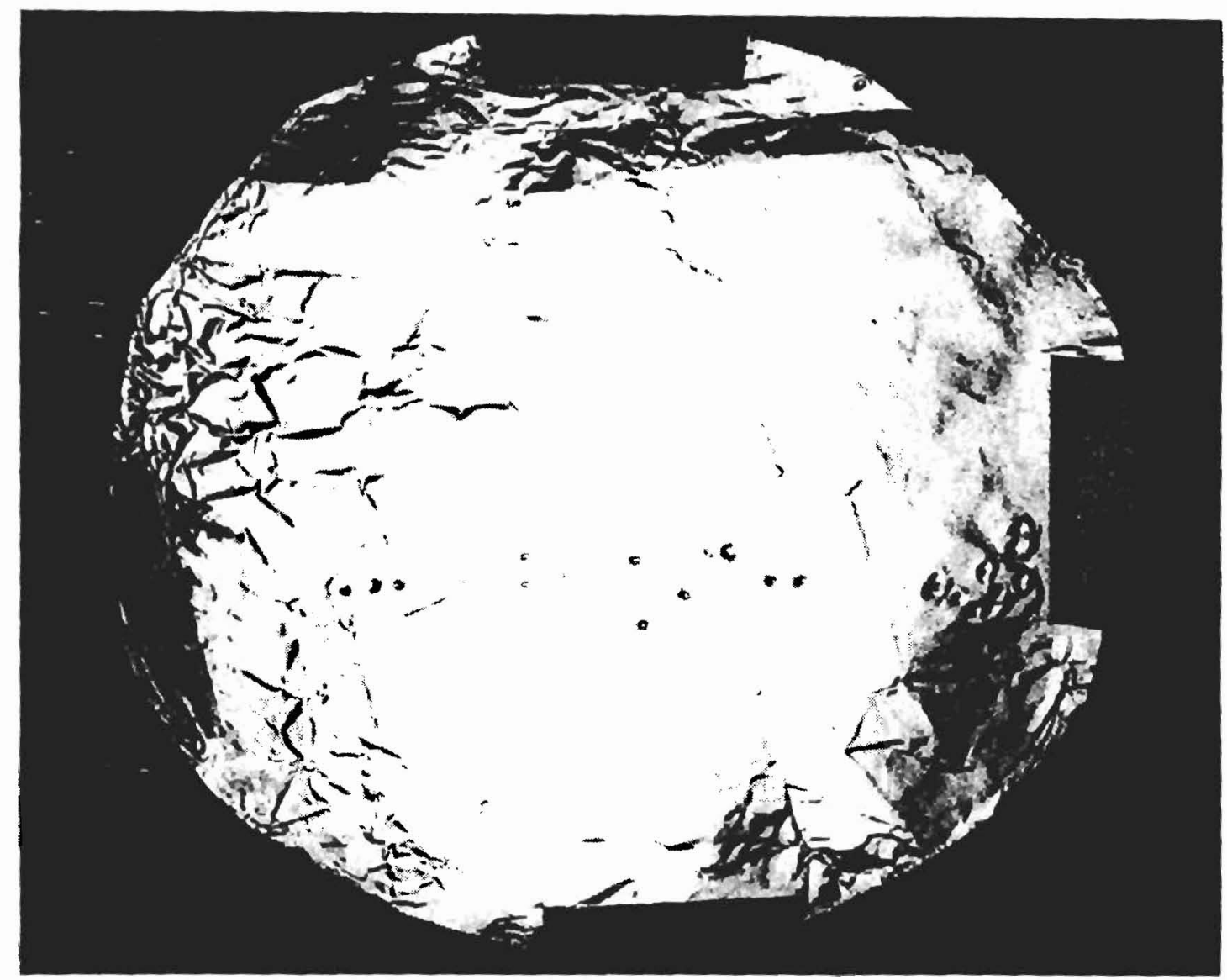

Fig. 2. Target foil showing angular spread in direction of the pellets leaving the guide tube under an improved launching method. 


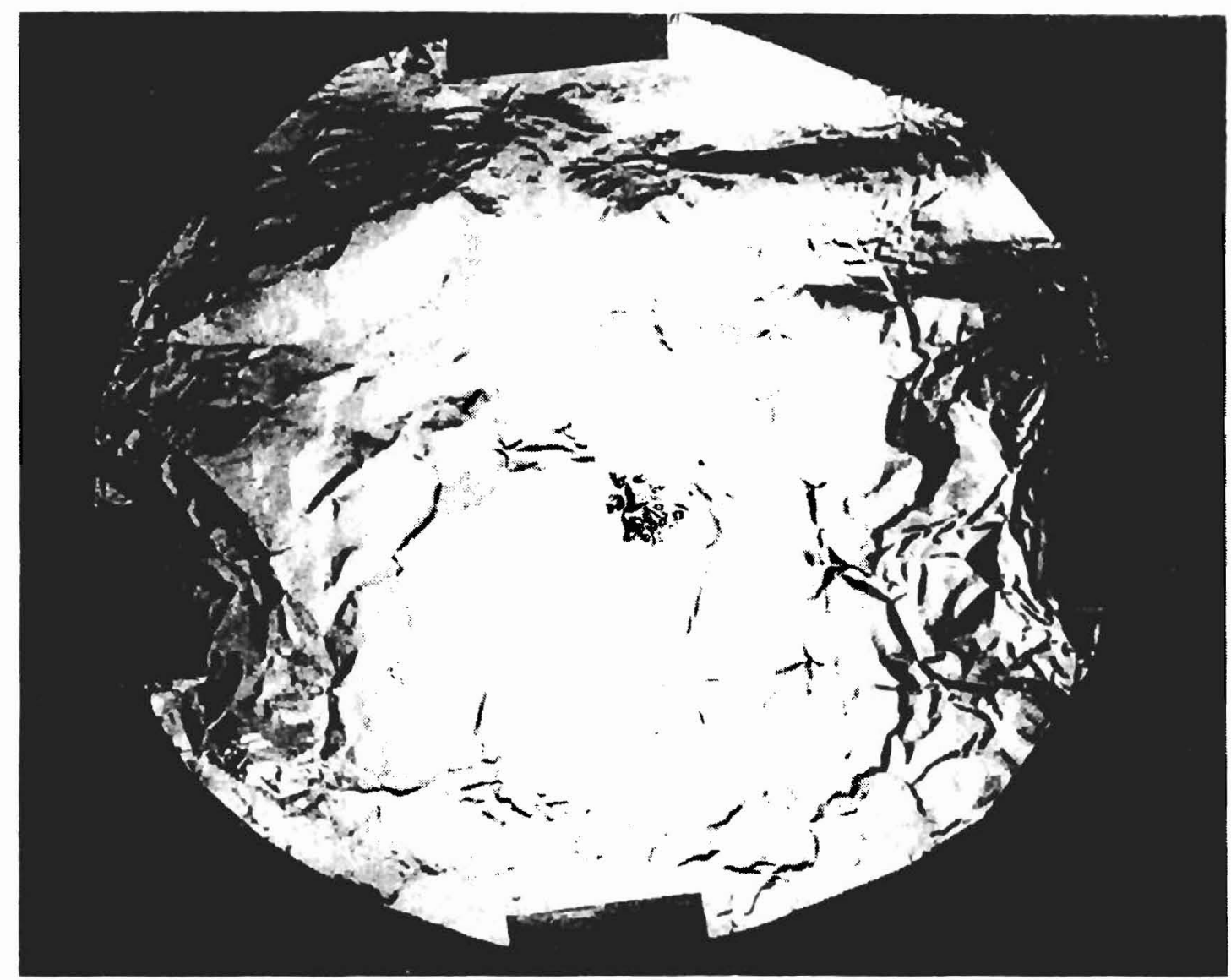

Fig. 3. Target foil showing the final angular spread in pellet direction on using the slide. 


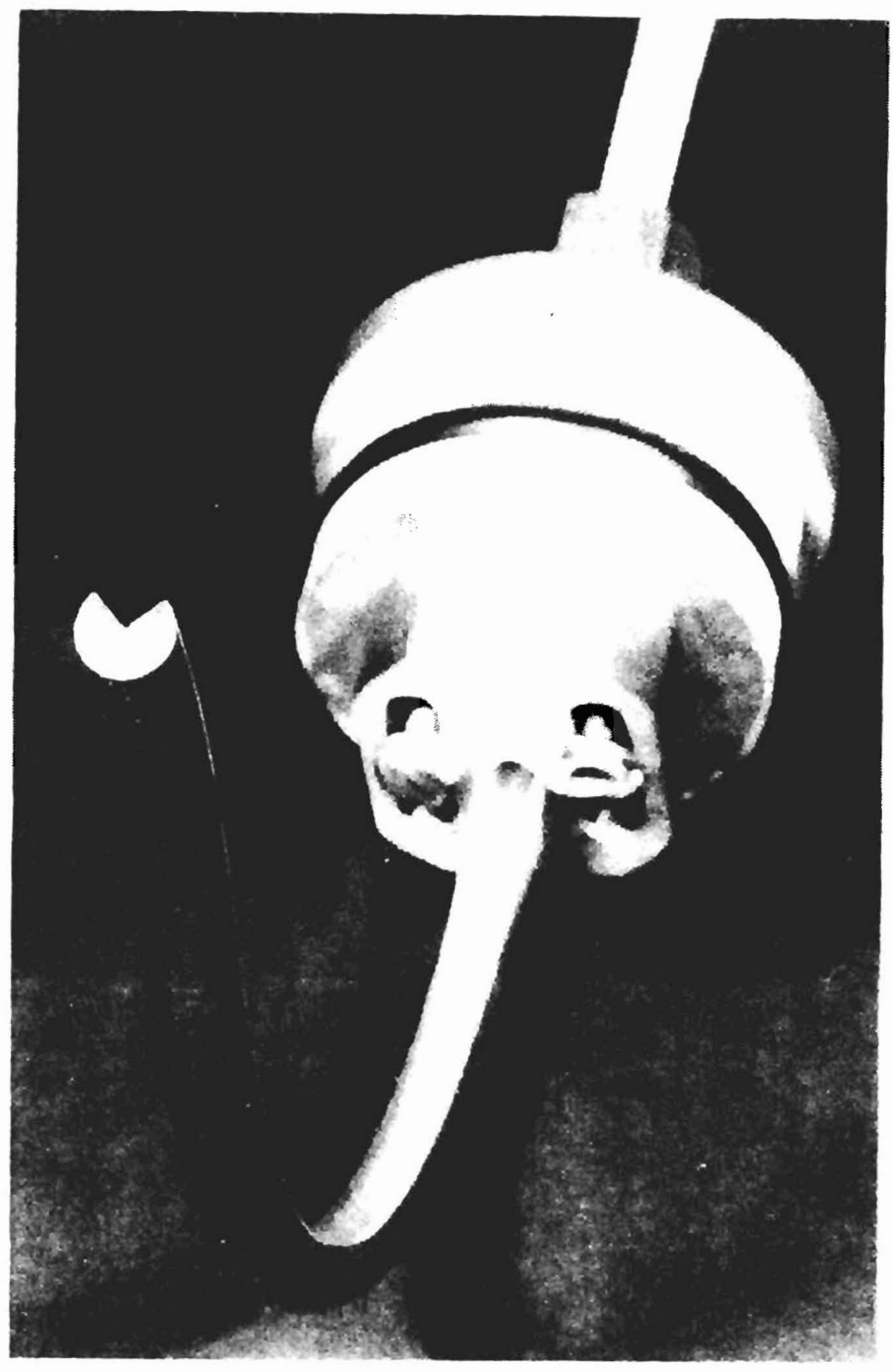

Fig. 4. The final version of the slide. 


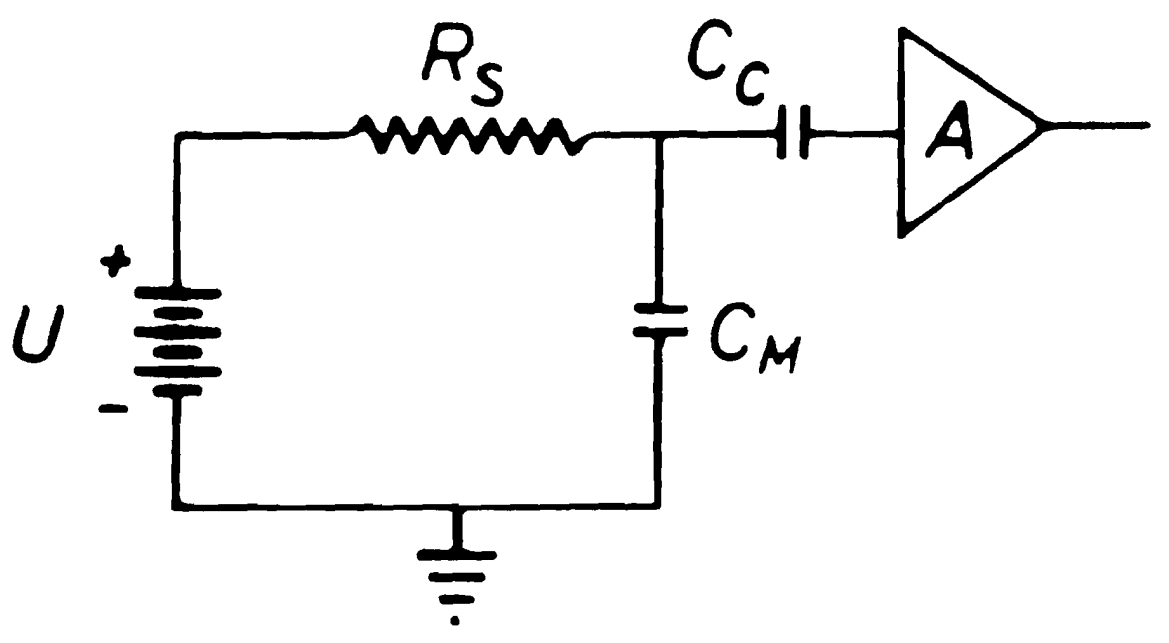

Fig. 5. Circuit for measuring the pellet size by the capacitor method.

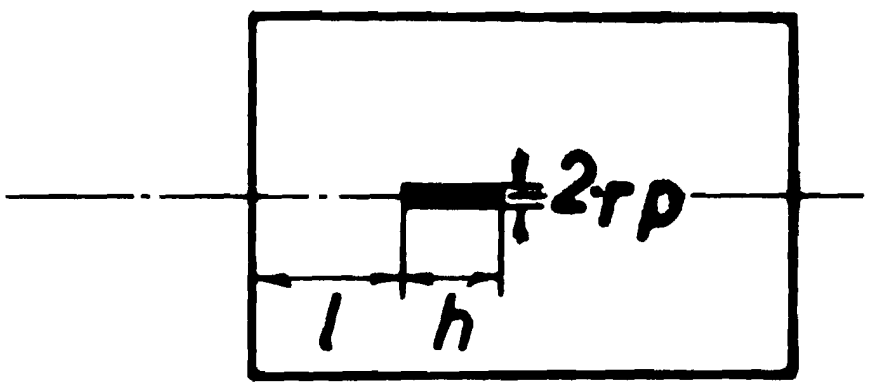

Fig. 6. Schematic drawing of pellet in the measuring microwave cavity. 


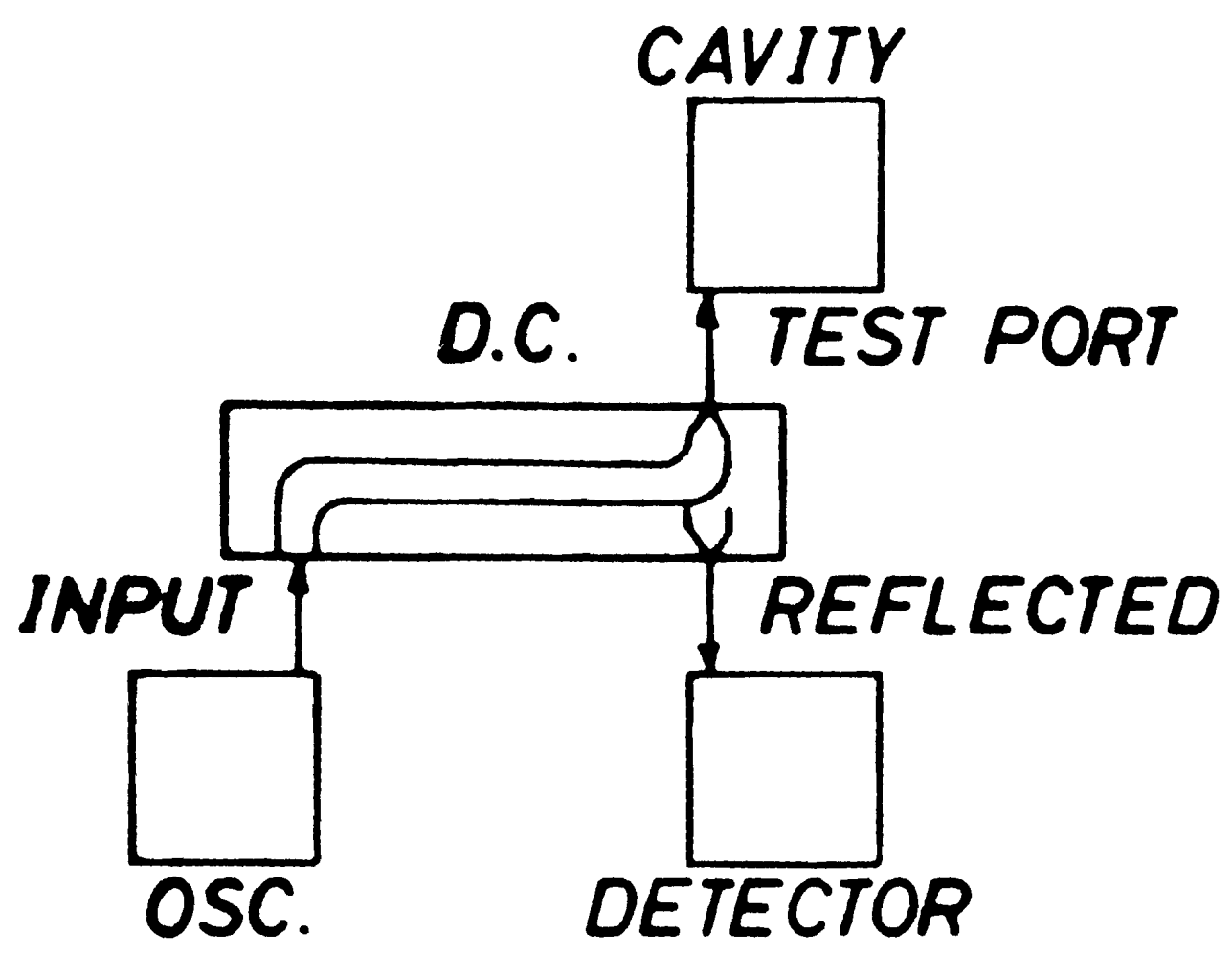

Fig. 7. Circuit for measuring the pellet size by the microwave method.

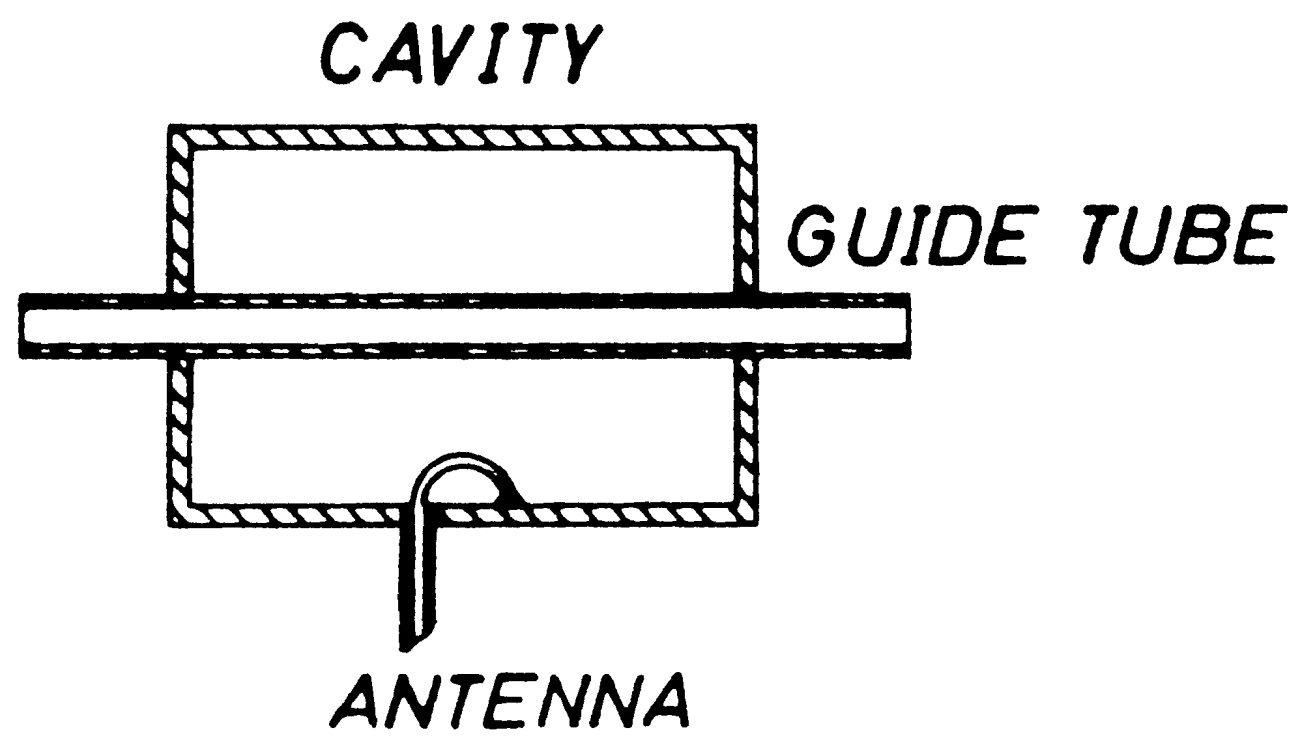

Fig. 8. Measuring cavity with mounted guide tube. 


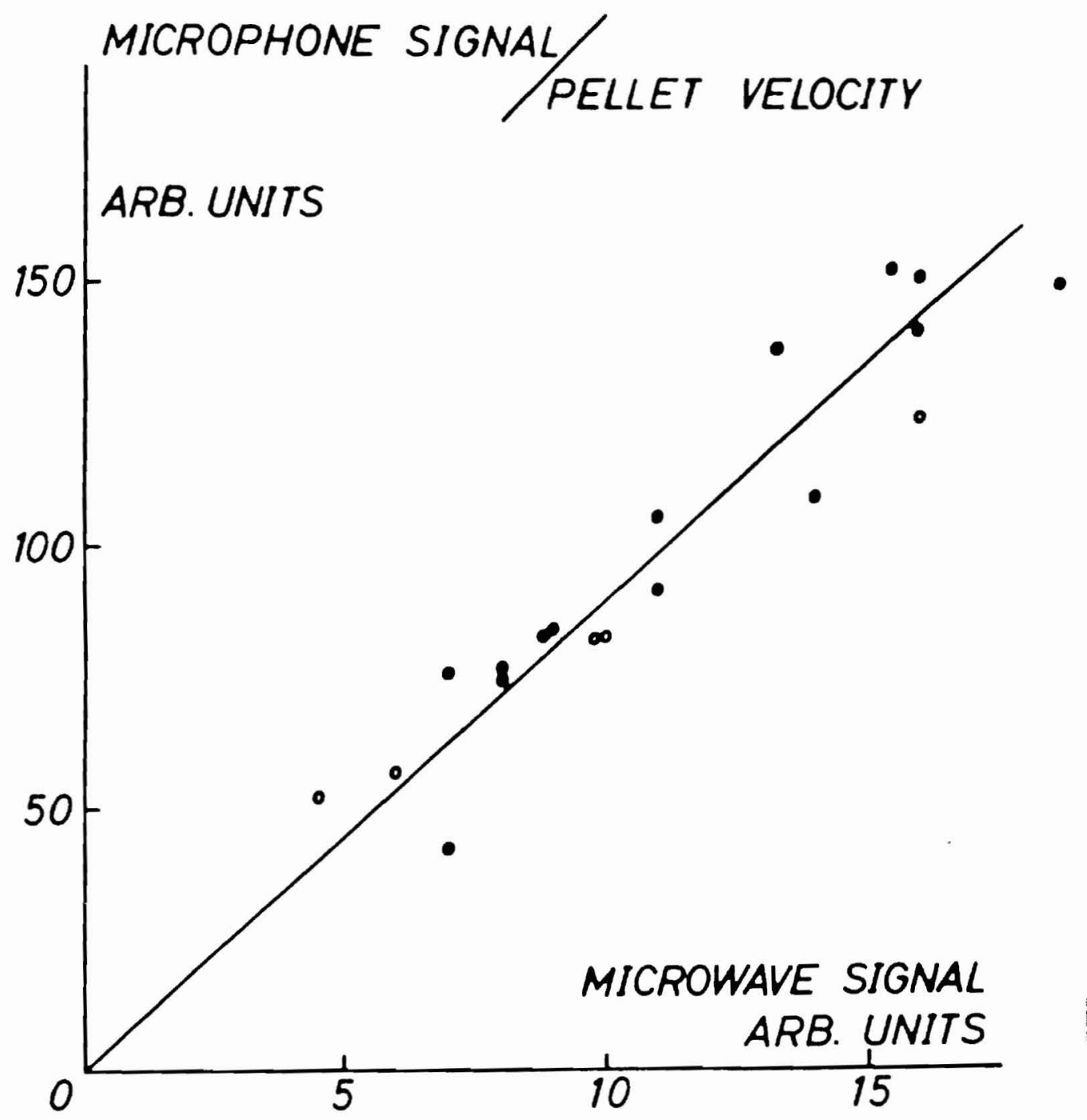

Fig. 9. Measurements of pellet mass by microphone signal vs. microwave cavity signal. 


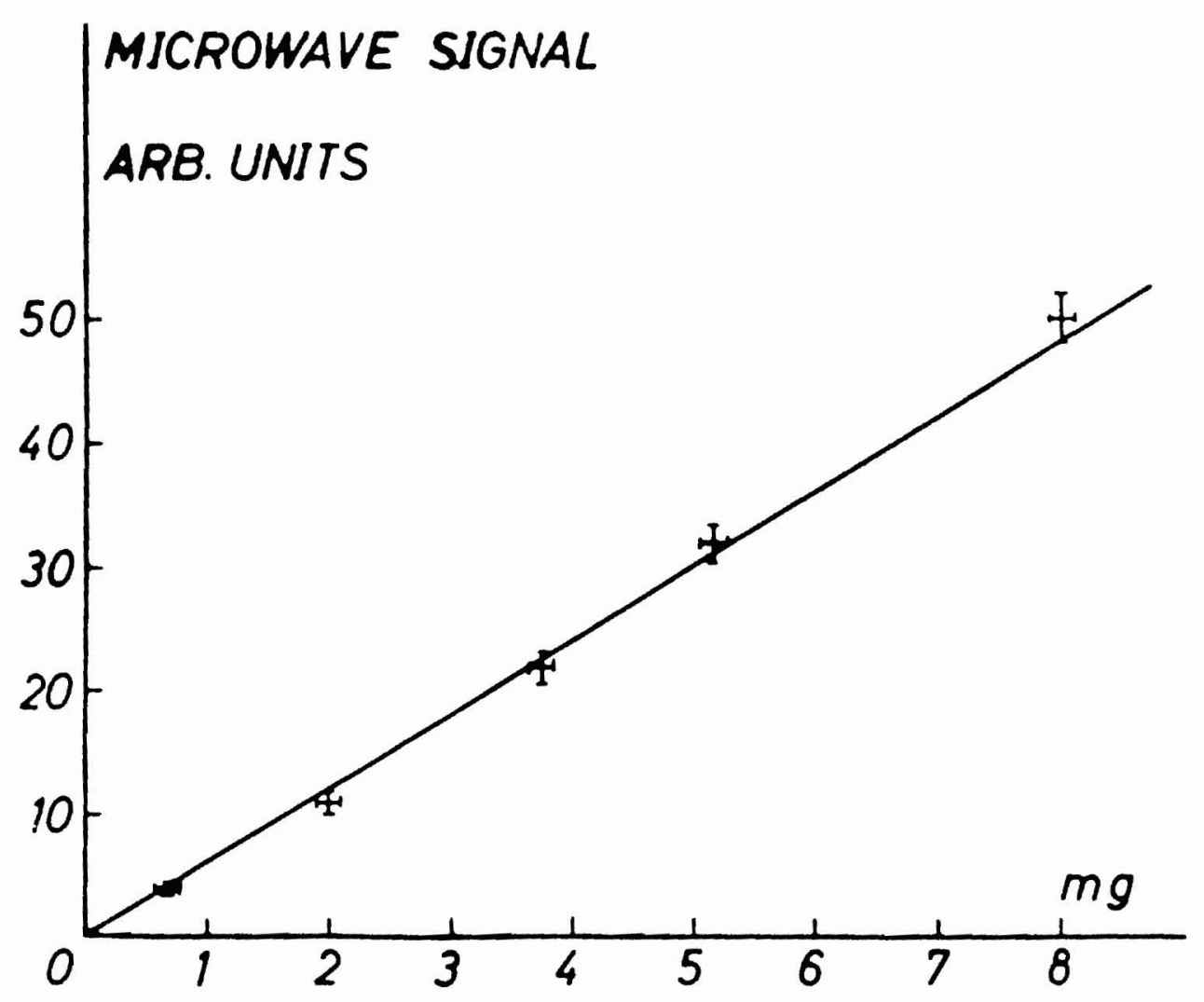

Fig. 10. Microwave signal strength vs, weight of dummy pellets. 
Handling of deuterium pellets for plasma refuelling

P.B. Jensen and V. Andersen

Department or group

Association

Euratom

The use of a guide tube technique to inject pellets in pellet-plasma experiments is described. The effect of the guide tube on the mass and speed of a slowly moving pellet $(\mathrm{v} \sim 150 \mathrm{~m} / \mathrm{s})$ is negligible. To improve the divergence in trajectories of the pellets on leaving the guide tube a specially formed brass slide has been developed, which improves the aiming accuracy by a factor of 10. A simple method for determining the mass of larger pellets by means of a plate capacitor through which the pellets are shot is described. A method for small pellets as well has been investigated. This scheme employs a microwave cavity operating at around $9 \mathrm{GHz}$.

Availa.jle on request from Ris $\phi$ Library, Ris $\varnothing$ National Laboratory (Ris $\varnothing$ Bibliotek), Fors $\varnothing$ gsanlæg Ris $\phi$ ), DK-4000 Roskilde, Denmark Telephone: (03) 371212 , ext. 2262. Telex: 43116 